\title{
Actitudes y estereotipos en estudiantes del área de la salud hacia personas con discapacidad motriz
}

\author{
Attitudes and stereotypes in students of the health area towards people \\ with motor disabilities
}

\begin{abstract}
Resumen
El objetivo central de esta investigación fue analizar las actitudes y los estereotipos hacia personas con discapacidad motriz en una muestra de estudiantes del área de la salud. Para ello se aplicaron la Escala Multidimensional de Actitudes Hacia Personas con Discapacidad y un instrumento para medir estereotipos (calidez y competencia) hacia personas con discapacidad. Se trabajó con 232 estudiantes (edad promedio: 23 años, DT=5), a quienes se les aplicaron los instrumentos en línea. Los resultados del análisis factorial muestran una estructura teóricamente congruente y propiedades psicométricas adecuadas para ambos instrumentos. Los participantes reportaron actitudes positivas hacia las personas con discapacidad y percepciones similares de competencia hacia individuos con y sin discapacidad. Sin embargo, reportaron niveles significativamente más altos de calidez para las personas con discapacidad. Estos resultados sugieren una baja disposición a reportar estereotipos de incompetencia o desagrado hacia personas con discapacidad, pero una percepción compensatoria de mayor calidez.
\end{abstract}

\section{Palabras clave}

Actitudes, estereotipos, calidez, competencia, discapacidad, México.

\begin{abstract}
The main purpose of this research was to analyze the attitudes and stereotypes towards people with motor disabilities in a sample of students from the health area. To this end, the Multidimensional Scale of Attitudes towards Persons with Disabilities and an instrument for measuring stereotypes (warmth and competence) towards people with disabilities. We worked with 232 students (average age: 23 years old, SD = 5) to whom the online instruments were applied. The results of the factor analysis show a theoretically congruent structure and adequate psychometric properties for both instruments. Participants reported positive attitudes towards people with disabilities and similar perceptions of competence towards individuals with and without disabilities. However, they reported significantly higher levels of warmth for people with disabilities. These results suggest a low disposition to report stereotypes of incompetence or dislike towards people with disabilities, but a compensatory perception of greater warmth.
\end{abstract}

\section{Keywords}

Attitudes, stereotypes, warmth, competence, disability, Mexico.

\section{Susana X. Bárcena Gaona} $<$ s.barcena@campus.iztacala. unam.mx>

Universidad Nacional Autónoma de México. México

\section{Christian Enrique Cruz Torres \\ <cassiel.79@gmail.com>}

Universidad de Guanajuato. México

\section{Benjamin C. Jenkins}

\section{Charles}

<benjamin.jenkins@wnmu.edu>

Western New Mexico University. México

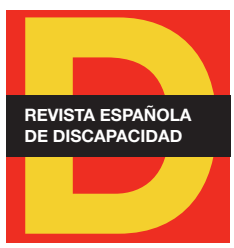

Para citar:

Bárcena, S. X. et al. (2018): "Actitudes y estereotipos en estudiantes del área de la salud hacia personas con discapacidad motriz". Revista Española de Discapacidad, 6 (I): 199219.

Doi: <https://doi.org/10.5569/23405104.06.01.10>

Fecha de recepción: 08-09-2017 Fecha de aceptación: 16-02-2018 


\section{Introducción}

El concepto de discapacidad propuesto por la Clasificación Internacional del Funcionamiento, de la Discapacidad y de la Salud (OMS, 2001) establece que las personas con discapacidad son aquellas que tienen una o más deficiencias físicas, mentales, intelectuales o sensoriales, las cuales pueden impedir su participación plena y efectiva en igualdad de condiciones a los demás en la interacción en distintos ambientes del entorno social. Desde esta perspectiva, la discapacidad no reside únicamente en el individuo, sino que se reconoce la importancia del medio social, al favorecer o limitar sus oportunidades de interacción dentro de un ambiente social. Por ello, "las personas con discapacidad viven discapacitadas por la sociedad y no sólo por su cuerpo" (OMS, 2015).

De acuerdo con el Informe Mundial de Discapacidad (OMS, 2011), en el mundo viven más de mil millones de personas con alguna discapacidad. Esta cifra representa alrededor del $15 \%$ de la población a nivel mundial. En dicho informe se establece que las personas con discapacidad tienen dificultades para acceder a la educación, el empleo, la atención de la salud y la participación social debido a las creencias y prejuicios por parte de la sociedad. Es decir, estas personas tienen menos probabilidades que sus homólogos sin discapacidad de ingresar en la escuela, permanecer en ella y superar los cursos sucesivos. De igual forma, los empleadores consideran, erróneamente, que son menos productivas y desconocen los ajustes disponibles para su inserción laboral. Así, las personas con discapacidad tienen más probabilidades de permanecer desempleadas y, cuando consiguen un empleo, perciben salarios bajos. En consecuencia, presentan tasas más altas de pobreza que las personas sin discapacidad.

En México viven 5.739.270 personas con algún tipo de discapacidad. Dicha cifra corresponde al 5,1\% de la población total del país. La discapacidad motriz es la que se presenta con mayor frecuencia (alrededor de la mitad de las limitaciones de las personas con discapacidad tienen que ver con dificultades para caminar o moverse). De las personas que tienen entre 3 y 29 años de edad y que viven con discapacidad motriz, únicamente el 10,9 \% asiste a la escuela. De la población con discapacidad mayor a 15 años, el 39,1\% participa en actividades económicas, en comparación con el 64,7 \% del mismo grupo etario que vive sin una discapacidad (INEGI, 2015).

La primera Encuesta Nacional sobre Discriminación en México (ENADIS) se llevó a cabo en 2005 y expuso que las personas con discapacidad constituyen uno de los once grupos más discriminados del país. En su edición más reciente del año 2010, se obtuvo información de una muestra de personas con discapacidad; la mayoría de ellas (56,3\%) tenía dificultad para moverse o caminar. De los encuestados, un $95 \%$ vivían con su familia; para más de la mitad (52,3\%), sus ingresos provienen de sus padres y otros familiares, y únicamente el 19,1\% consideró que sus ingresos son suficientes para vivir. La gran mayoría dijo que es difícil obtener algún apoyo del gobierno por medio de los programas públicos. De las personas que respondieron la encuesta, el $60 \%$ considera que los servicios médicos que recibe no son suficientes, debido a que no cuentan con la atención médica necesaria. De las personas con discapacidad motriz, el 47,3\% señala que en su ciudad no hay rampas para sillas de ruedas y el $65 \%$ no cuenta con baños con este tipo de accesos. Según ellas, el desempleo es el principal problema que enfrentan, seguido de la discriminación (Conapred, 2012). Es posible suponer que ambos problemas estén íntimamente ligados pues, durante siglos, la discriminación hacia las personas con discapacidad ha repercutido de manera negativa en su vida y la de su familia (Mackelprang y Salsgiver, 1996). 
Un eje tradicional del estudio del prejuicio y la discriminación son las actitudes. En la tabla 1 se presenta un resumen de los principales instrumentos que se han utilizado para estudiar este tema. La escala de Actitud para Personas Discapacitadas (ATDP) de Yuker et al. (1960) ha sido la más utilizada en las últimas décadas (Antonak y Livneh, 1988, 2000; Díaz y Quiroga, 2007). Esta escala fue diseñada para medir unidimensionalmente las actitudes hacia las personas con discapacidad. Posteriormente, Voeltz (1980) desarrolló una escala para medir las actitudes de los niños en la escuela primaria hacia compañeros con discapacidad que se estaban integrando al aula por primera vez en la historia de Estados Unidos. Esta escala fue considerada multidimensional por su creador, ya que se indagaban varios aspectos relacionados con la percepción hacia individuos con diferentes tipos de discapacidad. Sin embargo, Antonak y Livneh (1988) identificaron que algunas frases de esta escala (como "Tengo un amigo retrasado" o "Me gustaría ser amigo de un niño discapacitado") hacen referencia a la discapacidad de una manera ambigua, particularmente para los niños pequeños. Del mismo modo, la Escala de Actitudes hacia Personas Discapacitadas (SADP, por sus siglas en inglés) de Antonak (1982) también midió las actitudes hacia las personas con discapacidad como grupo general.

Este tipo de medición de las actitudes fue criticado por Siller, quien consideraba que no sólo las actitudes son constructos multidimensionales, sino que también lo es la discapacidad. Él desarrolló una variedad de escalas multidimensionales conocidas como Escalas de Factor de Discapacidad (DFS, por sus siglas en inglés). Las DFS miden las actitudes hacia individuos con discapacidades y condiciones de salud específicas que incluyen: amputación, ceguera y condiciones cosméticas (Siller et al., 1967a; Siller et al., 1967b), sordera (Ferguson, 1970), obesidad (Vann, 1970) y cáncer (Siller y Braden, 1976). Aunque las DFS fueron innovadoras en su intento de comprender cómo se percibe a las personas con discapacidades y condiciones de salud específicas, así como en su concepción de las actitudes como una construcción multidimensional, son criticadas por ser mínimamente publicadas y, por lo tanto, carentes de replicación independiente de sus estudios (Antonak y Livneh, 1988).

A partir de entonces, las mediciones de las actitudes hacia las personas con discapacidad se han vuelto más diversas. Por ejemplo, Tringo (1970) buscó comprender las actitudes y los prejuicios hacia una variedad de grupos de discapacidad con el desarrollo de la Escala de Distancias de Discapacidad Social (DSDS, por sus siglas en inglés). Tringo conceptualizó la discapacidad como una construcción multifacética y teorizó que las actitudes de las personas variaban dependiendo del tipo de discapacidad. La escala enumera 21 tipos diferentes de discapacidad y enfermedades crónicas, con lo cual los encuestados pueden indicar su grado de aceptación o proximidad social a una persona con cada condición. De manera similar, Grand et al. (1982) exploraron la aceptación social hacia las personas con parálisis cerebral, epilepsia, amputaciones y ceguera en el desarrollo de la Escala de Relación Social por Discapacidad (DSRS, por sus siglas en inglés). Yuker y Hurley (1987) examinaron el efecto que tendría sobre las actitudes la frecuencia del contacto con personas con discapacidad. Gething y Wheeler (1992) midieron cómo de cómodas se sentirían las personas en ciertas situaciones con personas con diversas discapacidades en su Escala de Interacción con Personas Discapacitadas (IDP, por sus siglas en inglés). Por último, Christison et al. (2002) desarrollaron la Escala de la Condición Médica (MCRS, por sus siglas en inglés), para medir cómo se sienten las personas (especialmente, los profesionales de la salud) cuando se enfrentan a personas que padecen diversas afecciones médicas o que tienen algún tipo de discapacidad.

Estas escalas han contribuido en gran medida a la comprensión actual de las actitudes hacia las personas con discapacidad. Sin embargo, carecen de sofisticación teórica y de un contexto situacional respecto a 
los grupos minoritarios y las personas con discapacidad (Antonak y Livneh, 2000). Por ello, Findler et al. (2007) contemplaron estos aspectos para desarrollar una escala multidimensional que permita obtener información de los componentes afectivos, cognitivos y conductuales de la actitud, para lo cual, propusieron la Escala de Actitudes Multidimensionales para Personas con Discapacidad (MAS, por sus siglas en inglés). Ésta busca disminuir la deseabilidad social en las respuestas de los participantes, al presentar situaciones hipotéticas para obtener información indirecta de las actitudes. Dicha escala fue traducida al español y validada en población colombiana, y ha sido utilizada en la presente investigación por los motivos ya referidos (Stevens et al., 2013).

\begin{tabular}{|c|c|c|c|}
\hline Instrumento y autor & $\begin{array}{l}\text { Escala de calificación } \\
\text { (reactivos) }\end{array}$ & Descripción de la escala & Confiabilidad \\
\hline $\begin{array}{l}\text { Attitudes Toward Disabled } \\
\text { Persons Scale (ATDP) } \\
\text { (Yuker et al., 1966) }^{\mathrm{a}}\end{array}$ & $\begin{array}{l}-3 \text { = no estar de acuerdo a }+3 \\
=\text { estar muy de acuerdo }(20 \\
\text { a } 30 \text { reactivos en diferentes } \\
\text { versiones) }\end{array}$ & $\begin{array}{l}\text { Escala unidimensional que mide } \\
\text { la actitud generalizada hacia las } \\
\text { personas con discapacidades físicas } \\
\text { como grupo. }\end{array}$ & Test/Retest 0.71-0.83 \\
\hline $\begin{array}{l}\text { Disability Factor Scale - } \\
\text { General (DFS-G) (Siller et } \\
\text { al., 1967) }\end{array}$ & $\begin{array}{l}1=\text { muy de acuerdo a } 6= \\
\text { totalmente en desacuerdo ( } 69 \\
\text { reactivos) }\end{array}$ & $\begin{array}{l}\text { Escala multidimensional compuesta } \\
\text { por } 7 \text { subescalas (por ejemplo, } \\
\text { tensión de interacción y rechazo } \\
\text { a la intimidad) que miden las } \\
\text { actitudes hacia las personas con } \\
\text { diversas discapacidades físicas y } \\
\text { enfermedades crónicas. }\end{array}$ & Cronbach $\alpha$ 0.73-0.87 \\
\hline $\begin{array}{l}\text { Disability Social Distance } \\
\text { Scale (DSDS) (Tringo, } \\
1970)^{a}\end{array}$ & $\begin{array}{l}\text { Escala de proximidad social } \\
\text { Bogardus-tipo de } 9 \text { niveles, } \\
1 \text { = se casaría con ella a } 9 \\
=\text { desearía que muera ( } 1 \\
\text { reactivo) }\end{array}$ & $\begin{array}{l}\text { Escala unidimensional que mide } \\
\text { las actitudes hacia las personas } \\
\text { con discapacidades específicas al } \\
\text { establecer la existencia y composición } \\
\text { de una jerarquía de preferencia sobre } \\
21 \text { discapacidades. }\end{array}$ & Cronbach $\alpha$ 0.95-0.98 \\
\hline $\begin{array}{l}\text { Acceptance Scale } \\
(\text { A-Scale) (Voeltz, 1980) }\end{array}$ & $\begin{array}{l}2=\text { aceptar respuesta, } 1= \\
\text { indeciso, } 0=\text { no aceptar }(22 \\
\text { a } 37 \text { reactivos en diferentes } \\
\text { versiones) }\end{array}$ & $\begin{array}{l}\text { Escala multidimensional compuesta } \\
\text { por } 4 \text { subescalas (por ejemplo: } \\
\text { tipos de contacto real y disposición } \\
\text { de contacto social) que miden las } \\
\text { actitudes hacia los compañeros con } \\
\text { discapacidades físicas integradas en } \\
\text { el aula regular. }\end{array}$ & $\begin{array}{l}\text { Cronbach } \alpha 0.77 \\
\text { Mitades partidas } 0.82\end{array}$ \\
\hline $\begin{array}{l}\text { Scale of Attitudes Toward } \\
\text { Disabled Persons (SADP) } \\
\text { (Antonak, 1982)a }^{a}\end{array}$ & $\begin{array}{l}-3=\text { no estar de acuerdo a }+3 \\
=\text { estar muy de acuerdo ( } 24 \\
\text { reactivos) }\end{array}$ & $\begin{array}{l}\text { Escala multidimensional compuesta } \\
\text { de } 3 \text { subescalas (Optimismo- } \\
\text { Derechos humanos, Conceptos } \\
\text { erróneos conductuales, Pesimismo- } \\
\text { Desesperanza) que miden las } \\
\text { actitudes hacia las personas con } \\
\text { discapacidad como grupo. }\end{array}$ & Cronbach $\alpha$ 0.74-0.91 \\
\hline $\begin{array}{l}\text { Disability Social } \\
\text { Relationships Scale } \\
\text { (DSRS) (Grand et al., } \\
\text { 1982) }\end{array}$ & $\begin{array}{l}\text { Verdadero o falso (18 } \\
\text { reactivos) }\end{array}$ & $\begin{array}{l}\text { Escala multidimensional que mide } \\
\text { la influencia de los factores de la } \\
\text { situación social en las actitudes hacia } \\
\text { las personas con parálisis cerebral, } \\
\text { epilepsia, amputación del brazo y } \\
\text { ceguera. }\end{array}$ & Cronbach $\alpha$ 0.86-0.95 \\
\hline
\end{tabular}




\begin{tabular}{|c|c|c|c|}
\hline Instrumento y autor & $\begin{array}{l}\text { Escala de calificación } \\
\text { (reactivos) }\end{array}$ & Descripción de la escala & Confiabilidad \\
\hline $\begin{array}{l}\text { Contact with Disabled } \\
\text { Persons Scale (CDP) } \\
\text { (Yuker y Hurley, 1987) }\end{array}$ & $\begin{array}{l}\text { Escala de } 5 \text { puntos, } 1=\text { nunca } \\
\text { a } 5=\text { muy a menudo ( } 20 \\
\text { elementos). }\end{array}$ & $\begin{array}{l}\text { Escala multidimensional que mide } \\
\text { la frecuencia de contacto y la } \\
\text { experiencia que una persona ha } \\
\text { tenido con una persona con una } \\
\text { discapacidad. }\end{array}$ & Cronbach $\alpha$ 0.89-0.92 \\
\hline $\begin{array}{l}\text { Issues in Disability Scale } \\
\text { (IDS) (Makas et al., 1988) }^{\text {a }}\end{array}$ & $\begin{array}{l}7=\text { muy de acuerdo a } 1= \\
\text { totalmente en desacuerdo (55 } \\
\text { reactivos). }\end{array}$ & $\begin{array}{l}\text { Escala multidimensional compuesta } \\
\text { por } 6 \text { subescalas (Educación, } \\
\text { Legal, Íntimo Social, Social no } \\
\text { Íntimo, Habilidades Fisiológicas, } \\
\text { Características Psicológicas) que } \\
\text { miden las actitudes hacia las personas } \\
\text { con diversas discapacidades físicas } \\
\text { y las personas con discapacidad en } \\
\text { general. }\end{array}$ & Cronbach $\alpha 0.86$ \\
\hline $\begin{array}{l}\text { Interaction with Disabled } \\
\text { Persons Scale (IDP) } \\
\text { (Gething y Wheeler, 1992) }^{a}\end{array}$ & $\begin{array}{l}\text { Escala de } 6 \text { puntos } 6=\text { muy } \\
\text { de acuerdo a } 1: \text { totalmente en } \\
\text { desacuerdo ( } 20 \text { reactivos). }\end{array}$ & $\begin{array}{l}\text { Escala multidimensional compuesta } \\
\text { de } 6 \text { subescalas (por ejemplo, } \\
\text { vulnerabilidad, afrontamiento, nivel } \\
\text { de información percibido) que mide } \\
\text { la incomodidad en las interacciones } \\
\text { sociales con personas con } \\
\text { discapacidad. }\end{array}$ & Cronbach $\alpha$ 0.54-0.86 \\
\hline $\begin{array}{l}\text { Medical Condition Regard } \\
\text { Scale (MCRS) (Christison } \\
\text { et al., 2002) }\end{array}$ & $\begin{array}{l}\text { Escala de } 6 \text { puntos, } 1= \\
\text { totalmente en desacuerdo a } 6 \\
=\text { totalmente de acuerdo ( } 18 \\
\text { reactivos) }\end{array}$ & $\begin{array}{l}\text { Escala multidimensional que mide } \\
\text { los sesgos positivos y negativos, } \\
\text { las emociones y las expectativas } \\
\text { producidas por } 12 \text { descriptores de la } \\
\text { condición médica. }\end{array}$ & $\begin{array}{l}\text { Cronbach } \alpha 0.87 \\
\text { Test/Retest } 0.84\end{array}$ \\
\hline $\begin{array}{l}\text { Multidimensional Attitudes } \\
\text { Scale Toward Persons } \\
\text { with Disabilities (MAS) } \\
\text { (Findler et al., 2007). }\end{array}$ & $\begin{array}{l}\text { Escala de } 5 \text { puntos, } 1=\text { de } \\
\text { ningún modo a } 5=\text { muchísimo } \\
\text { (34 reactivos) }\end{array}$ & $\begin{array}{l}\text { Escala multidimensional compuesta } \\
\text { de } 3 \text { subescalas (afectiva, cognitiva y } \\
\text { comportamiento) que mide actitudes } \\
\text { explícitas hacia personas con } \\
\text { discapacidad motriz. }\end{array}$ & Cronbach $\alpha$ 0.68- 0.90 \\
\hline
\end{tabular}

a Estas escalas han sido revisadas por Antonak y Livneh (1988). Fuente: elaboración propia.

En una gran cantidad de los estudios publicados en idioma inglés sobre actitudes se cuenta con la participación de estudiantes universitarios, principalmente aquellos cuyas profesiones están vinculadas con las ciencias de la salud y se debe, sobre todo, a su fácil acceso y disposición (Goodwin y Goodwin, 2017). No obstante, su estudio resulta fundamental si se considera que son ellos los que, de manera directa o indirecta, estarán en contacto con este colectivo y tendrán la encomienda de procurar su cuidado, rehabilitación e inclusión social. Los resultados más importantes de estas investigaciones indican que las mujeres, en comparación con los hombres, tienen actitudes más positivas hacia las personas con discapacidad (Hampton y Xiao, 2007). Por ejemplo, Tervo et al. (2002) encontraron que las mujeres, en comparación con los hombres, están en desacuerdo con que los programas de rehabilitación son demasiado costosos. En cuanto al tipo de programas, los estudiantes de recreación (Hodge et al.,1998), sociología, educación y trabajo social (Martínez et al., 2011), en comparación con otras áreas, expresan actitudes más favorables hacia las personas con discapacidad. Asimismo, los individuos que tuvieron contacto previo con estas personas muestran conductas más favorables hacia ellas que quienes no lo tuvieron (Satchidanand et al., 2012). 
En un estudio experimental se compararon las actitudes hacia las personas con discapacidad entre dos grupos de estudiantes, se les aplicó la Escala Multidimensional de Actitudes Hacia Personas con Discapacidad al inicio y al final del semestre, uno de los grupos recibió un curso sobre discapacidad y el otro un curso regular de psicología. Al comparar las puntuaciones en la escala al término del curso, no hubo diferencias significativas entre ambos grupos, los dos tenían actitudes positivas antes y después del curso. Un dato que resulta relevante es que un porcentaje cercano al $80 \%$ de los participantes había tenido alguna vez un compañero con discapacidad en clase. Es probable suponer que fue esta variable y no el curso lo que pudo influir en generar actitudes positivas en todos participantes de esta investigación (Meyers y Lester, 2016).

En estudios efectuados en España que analizan la temática de las actitudes en estudiantes del área de la salud se emplea consistentemente la Escala de Actitudes hacia Personas con Discapacidad. Forma G, desarrollada por Verdugo et al. (1994). Dicho instrumento está compuesto por 37 reactivos que conforman los siguientes factores: Factor 1: Valoración de limitaciones y capacidades, Factor 2: Reconocimiento/Negación de derechos, Factor 3: Implicación personal, Factor 4: Calificación genérica y Factor 5: Asunción de roles. La persona evaluada debe responder si está de acuerdo o no con cada una de las frases que se le presentan (escala de seis puntos). La escala cuenta con propiedades psicométricas adecuadas en términos de validez y confiabilidad.

Los resultados arrojados por dicha escala en una investigación llevada a cabo por Polo et al. (2011), en un grupo de estudiantes de Ciencias Sociales y Psicología, muestran actitudes positivas hacia las personas con discapacidad en cada uno de los factores, sobre todo en la implicación personal, ya que los encuestados presentan una predisposición favorable para relacionarse socialmente con una persona con discapacidad y rechazan su reclusión social. Asimismo, los estudiantes de psicología, en comparación con los otros estudiantes, obtuvieron la puntuación más alta en el factor "Calificación genérica". También quienes mencionaron haber tenido contacto previo con este colectivo mostraron actitudes más positivas. El motivo de contacto más frecuente de los encuestados obedecía a una relación familiar con la persona con discapacidad. Es probable que las actitudes positivas expresadas estén vinculadas con el contacto cercano y profundo característico de las relaciones familiares. Aunque no se presenta un análisis comparativo de las actitudes a partir del género (hombre/mujer) de los participantes, en este estudio la mayoría de los participantes eran mujeres. Es posible que esta variable intervenga tanto en la elección de carrera como en el tipo de actitudes, tal y como se demuestra en otras investigaciones donde las mujeres tienen mejores actitudes hacia las personas con discapacidad (Novo-Corti et al., 2015).

Moreno et al. (2006) aplicaron la escala referida en el estudio anterior a 498 estudiantes matriculados en carreras relacionadas con la discapacidad (magisterio, psicopedagogía y psicología). Los resultados muestran que los estudiantes poseen actitudes positivas hacia las personas con discapacidad y son más positivas en quienes han tenido contacto previo con estas personas. A diferencia del estudio anterior, no se encontraron diferencias por el tipo de curso ni tampoco por el género (hombre/mujer).

En contraste, en un estudio llevado a cabo con 258 estudiantes de siete escuelas de la Facultad de Medicina de la Universidad de Chile, se aplicó la encuesta ya referida y se encontraron diferencias en las actitudes de los estudiantes en función de las escuelas. Los estudiantes de la Escuela de Terapia Ocupacional alcanzaron puntuaciones que indican actitudes positivas en los factores "Implicación social", "Reconocimiento/negación de derechos" y "Asunción de roles". En cambio, los de las escuelas de Fonoaudiología y Nutrición presentaron una tendencia a calificar negativamente a las personas con discapacidad (Mella y González, 2007). 
Los estudios mencionados, aunque resultan valiosos, pueden generar respuestas sesgadas, debido al tipo de medición, puesto que las personas pueden sentirse menos dispuestas a reconocer sus actitudes negativas hacia un grupo socialmente vulnerable, como son las personas con discapacidad (Carver et al. 1978; Feinberg, 1967; Marini, 2012). Además, es necesario considerar que la discriminación puede presentarse en otros ejes de análisis. Por ejemplo, Boyle et al. (2010) encuestaron a 584 estudiantes de carreras afines al área de la salud para conocer sus actitudes hacia pacientes con discapacidad intelectual y encontraron que, en general, muestran una respuesta positiva hacia ellos. No obstante, puntuaron alto en el grado de acuerdo en afirmaciones como: "Tratar este tipo de pacientes es un desperdicio de dólares", "Los pacientes de este tipo me irritan", "Es poco lo que puedo hacer por este tipo de pacientes" y "Siento especial compasión por este tipo de pacientes". Estas expresiones reflejan - además de su connotación valorativa- creencias que refieren una baja utilidad social y poca capacidad de acción de las personas con discapacidad. Para entender estas dimensiones - que se configuran en creencias adicionales a las valoraciones actitudinales- es necesario entender la estructura del contenido de los estereotipos, además de su valoración. Al respecto, Fiske et al. (2002) proponen un modelo de análisis de los estereotipos mediante los ejes de calidez (ejemplo: amabilidad, tener buenos sentimientos) y competencia (ejemplo: eficiente, hábil). De esta manera, los grupos pueden ser percibidos no sólo de forma negativa o positiva, sino con estereotipos que les asignan ciertos roles de acuerdo con sus atributos de calidez y competencia.

Los integrantes de grupos sociales de bajo estatus a menudo son estereotipados como menos competentes, pero la valoración afectiva hacia ellos depende de sus niveles de calidez, es decir, grupos con estereotipos de alta calidez (ejemplo: adultos mayores) reciben valoraciones afectivas positivas sin importar su baja competencia percibida. Por su parte, los integrantes de baja competencia y baja calidez (ejemplo: personas en condición de calle) reciben valoraciones afectivas negativas. Por su parte, los integrantes de grupos sociales de mayor estatus son evaluados también respecto a estas dimensiones. En este sentido, los grupos de alta competencia, pero baja calidez pueden ser percibidos como amenazas potenciales, mientras que la combinación alta competencia y calidez se reserva para el propio grupo. Así, por ejemplo, las personas con discapacidad podrían ser percibidas con características positivas, como tener buenos sentimientos y ser pacientes, amables o tolerantes, pero carentes de otros atributos necesarios para acceder a funciones sociales de mayor estatus, como ser competentes o hábiles.

Es posible suponer que en las escalas utilizadas para medir actitudes hacia las personas con discapacidad, la gente reporte actitudes positivas, debido a que las consideran cálidas, pero no componentes y, como se ha visto, esta idea refuerza los estereotipos de incompetencia hacia este colectivo. Debido a esta complejidad de los estereotipos y los procesos de discriminación en general, resulta necesario contar con mediciones complementarias, tanto de los prejuicios basados en actitudes como de los estereotipos, mediante los ejes de calidez y competencia.

Por lo anterior, esta investigación tuvo por objetivo analizar las actitudes existentes hacia personas con discapacidad en términos de valencia (positivo y negativo) y conocer sus estereotipos por medio de los ejes de calidez y competencia, los cuales se han identificado previamente como fundamentales para entender las relaciones de discriminación e inequidad social entre grupos. Para ello, se analizaron además la validez de constructo, criterio y consistencia interna de la Escala Multidimensional de Actitudes Hacia Personas con Discapacidad, adaptada y validada al español por Stevens et al. (2013) y la medición de rasgos de calidez y competencia de Fiske et al. (2002), adaptada en este caso mediante viñetas para su aplicación al este- 
reotipo de personas con discapacidad. También se llevó a cabo un análisis con el propósito de identificar el papel que desempeña el contacto previo en las actitudes. Como hipótesis se proponen las siguientes:

- Ambos instrumentos mostrarán estructuras factoriales congruentes con las teorías que los respaldan y niveles adecuados de consistencia interna, además de presentar correlatos positivos entre sí que dan cuenta de la congruencia teórica de ambas mediciones.

- Dado el perfil de la muestra, se espera que las actitudes y los estereotipos reportados hacia personas con discapacidad serán en general positivos, aunque con mayores puntajes en la dimensión de calidez hacia las personas con discapacidad en comparación con personas sin discapacidad.

- Se espera que las personas que han mantenido un contacto previo con una persona con discapacidad tendrán actitudes más positivas.

\section{Método}

\subsection{Participantes}

Se trabajó con una muestra no probabilística de 164 mujeres y 44 hombres. Se consideró como criterio de inclusión que los participantes fueran estudiantes de universidades públicas en México, de alguna carrera universitaria del área de la salud. De esta manera, la muestra estuvo constituida, principalmente, por estudiantes de las carreras de Psicología (68,8 \%), Medicina (8,7\%) Enfermería (8,7 \%) y otras (13,8\%). La edad promedio de los participantes fue de 23,18 años, con una $\mathrm{DT}=5.06$. Se les preguntó a los participantes si tenían algún tipo de discapacidad: sólo cuatro de ellos declararon tener alguna.

\subsection{Instrumentos}

Los participantes respondieron una traducción al español de la Escala Multidimensional de Actitudes Hacia Personas con Discapacidad (Stevens et al., 2013). En este instrumento se pide a los participantes imaginar que interactúan con una persona con discapacidad ${ }^{1}$ y se les pregunta cómo de probable es que experimenten un conjunto de emociones (14 reactivos), pensamientos (10 reactivos) y conductas (6 reactivos) en esa circunstancia. Los reactivos se presentan en un formato tipo Likert con opciones que van de 1 (nada) a 5 (muchísimo).

Los estereotipos de calidez y competencia se evaluaron mediante los instrumentos que diseñaron Fiske et al. (2002) para este propósito, mediante seis reactivos que miden competencia y seis más que evalúan calidez, presentados en formato tipo Likert con opciones que iban de 1 (casi nunca) a 5 (casi siempre). En estudios previos los reactivos de competencia se han agrupado en un solo factor con la obtención de

1. Imagina la siguiente situación: José fue a almorzar con un grupo de amigos a un restaurante. Una persona en silla de ruedas, a quién José no conoce, entra en la cafetería y se une al grupo. A José le presentan a esa persona y, poco después, todos sus amigos se van, dejando solos en la mesa a José y a la persona en silla de ruedas. José tiene que esperar 15 minutos para que lo recojan. Trate de imaginar la situación. 
niveles de consistencia interna de $\mathrm{a}=.94$, mientras que los reactivos de calidez se agrupan también en un solo factor con consistencia interna de $a=.90$ (Fiske et al., 2002). Estos reactivos se respondían respecto a dos viñetas que presentaban descripciones breves de personas, una de ellas con discapacidad ${ }^{2}$ y otra sin discapacidad ${ }^{3}$, y se solicitaba a los participantes que respondieran cómo se imaginaban que eran estas personas. Para controlar los efectos del sexo de los personajes, se utilizaron dos versiones diferentes de estas viñetas, con el objetivo de contrabalancear el sexo de los personajes. La mitad de los participantes respondió a viñetas donde la persona con discapacidad era hombre y la otra mitad a viñetas donde era mujer. Por un error de traducción, uno de los reactivos de la escala de competencia (confident) tuvo que ser descartado del análisis.

Por último, se les preguntó a los participantes si conocían a alguna persona con discapacidad, cuál era el parentesco o relación con esa persona y qué tipo de discapacidad tenía esa persona.

\subsection{Procedimiento}

Los instrumentos fueron presentados en una plataforma para ser resueltos en línea. Se invitó a los estudiantes de las distintas universidades a participar en el estudio mediante redes sociales, con la garantía de que sus respuestas serían confidenciales y que sólo se analizarían con fines de investigación científica. Las respuestas fueron recopiladas entre el 15 y el 24 de noviembre de 2016.

\section{Resultados}

En la primera parte de esta sección se presentan análisis que buscan poner a prueba y presentar evidencias de las propiedades psicométricas de los instrumentos utilizados. Lo anterior se considera necesario para este estudio puesto que estos instrumentos no se han utilizado previamente en participantes de México, por lo que resulta necesario ofrecer evidencias de su validez y confiabilidad para respaldar las pruebas de hipótesis posteriores. La segunda parte se dedica justo a los análisis para poner a prueba las hipótesis del estudio.

\subsection{Propiedades psicométricas de los instrumentos}

Para poner a prueba la validez de constructo de la Escala Multidimensional de Actitudes Hacia Personas con Discapacidad (EMAHPD) se sometió cada una de las tres escalas a análisis factoriales de máxima

2. Alonso tiene 33 años y trabaja desde hace cuatro para una empresa en su ciudad. Su trabajo le permite ganar suficiente dinero para cubrir sus gastos y darse algún gusto de vez en cuando. Para caminar debe utilizar unas muletas especiales todo el tiempo dado que, cuando era niño, tuvo una infección que le impide mover las piernas casi por completo. Cuando lo piensa, Alonso cree que esta enfermedad dejó una huella muy profunda en su vida.

3. Laura tiene 35 años y vive en una ciudad mediana, en una casa cómoda, pero sin lujos. Es empleada en una empresa local desde hace 5 años. Cuando tenía 10 años, el autobús en el que viajaba sufrió un accidente y a raíz de eso sufrió una fractura en las piernas, por lo que tuvo que usar una silla de ruedas por un tiempo. Laura considera que ese accidente marcó de manera muy importante su vida. 
verosimilitud, con rotación ortogonal varimax. Como criterio de extracción para los factores se consideró un valor propio superior a 1. La consistencia interna de cada escala se calculó mediante la fórmula alfa de Cronbach.

Aunque existen recomendaciones genéricas respecto al tamaño mínimo de una muestra para realizar un análisis factorial, criterios más recientes proponen que estas recomendaciones no tienen en cuenta muchas de las dinámicas complejas de un análisis de factores (Henson y Roberts, 2006), encontrándose que es muy complicado encontrar o establecer reglas generales sobre el tamaño de la muestra aplicables a cualquier análisis factorial (MacCallum et al. 2001). Siguiendo este enfoque, se decidió valorar si el tamaño de la muestra era adecuado para este análisis mediante el índice Kaiser-Meyer-Olkin de adecuación muestral, para el cual se consideran valores de .8 o superiores como adecuados para el uso del análisis factorial (Kaiser, 1970). Con un valor de $\mathrm{KMO}=.867$, se considera que el tamaño de la muestra fue suficiente para llevar a cabo este análisis. El resultado de la escala de emociones se muestra en la tabla 2, y refleja una estructura que convergió en seis rotaciones, lo que generó tres factores que, en conjunto, explican 53,61\% de la varianza, con valores de bondad de ajuste $\mathrm{Ji}^{2}=90.21, \mathrm{gl}=52, \mathrm{p}<.001$. El primer factor fue nombrado Prejuicio, por reunir reactivos que refieren emociones negativas que tienen como puntero al reactivo Rechazo. El segundo factor fue nombrado Tranquilidad, puesto que hace referencia a la sensación de ese estado ante la posibilidad de interactuar con alguien con discapacidad. El tercer factor fue nombrado Ansiedad, por hacer referencia a emociones de incomodidad ante la viñeta.

\section{Tabla 2. Estructura factorial de la escala de emociones}

\begin{tabular}{|l|c|c|c|}
\multicolumn{1}{c|}{} & Prejuicio & Tranquilidad & Ansiedad \\
\hline Alfa de Cronbach & 0.839 & 0.875 & 0.804 \\
\hline Varianza explicada & $22.16 \%$ & $16.18 \%$ & $15.26 \%$ \\
\hline Rechazo & .719 & -.253 & .186 \\
\hline Vergüenza & .701 & -.120 & .257 \\
\hline Miedo & .618 & -.223 & .229 \\
\hline Culpa & .587 & -.069 & .191 \\
\hline Impotencia & .563 & -.064 & .272 \\
\hline Molestia & .530 & -.064 & .329 \\
\hline Depresión & .528 & -.069 & .124 \\
\hline Serenidad & -.126 & .912 & -.158 \\
\hline Calma & -.185 & .814 & -.106 \\
\hline Relajación & -.126 & .702 & -.305 \\
\hline Nerviosismo & .286 & -.222 & .761 \\
\hline Tensión & .349 & -.150 & .736 \\
\hline Estrés & .424 & -.167 & .515 \\
\hline Timidez & .204 & -.181 & .484 \\
\hline
\end{tabular}

Fuente: elaboración propia. 
Para el análisis de los componentes cognitivos de la escala fue necesario retirar el reactivo "Es posible que nos llevemos bien", porque generaba una estructura factorial poco clara conceptualmente. La estructura resultante con los demás reactivos es de un solo factor y convergió en cuatro iteraciones, con indicadores de bondad de ajuste $\mathrm{Ji}^{2}=75.79, \mathrm{gl}=27, \mathrm{p}<.001$, lo cual se muestra en la tabla 3 . El valor $\mathrm{KMO}=.883$ indica que el tamaño de la muestra fue adecuado para este análisis.

\begin{tabular}{|c|c|}
\hline & Cognición \\
\hline Alfa de Cronbach & 0.859 \\
\hline Varianza explicada & $43.26 \%$ \\
\hline Él parece amigable. & .819 \\
\hline Él se ve como una buena persona. & .770 \\
\hline Él parece ser una persona interesante. & .720 \\
\hline Él disfrutará conocerme. & .716 \\
\hline Siempre puedo hablar con él sobre cosas que nos interesen a los dos. & .713 \\
\hline ¿Por qué no llegar a conocerlo mejor? & .596 \\
\hline Me gusta conocer gente nueva. & .550 \\
\hline Yo puedo hacer que él se sienta cómodo. & .502 \\
\hline Si yo inicio la conversación él me lo agradecerá. & .427 \\
\hline
\end{tabular}

Fuente: elaboración propia.

En el análisis de la escala de conductas fue necesario también descartar el reactivo "Continuar con lo que él estaba haciendo", puesto que su carga factorial en esa única dimensión es inferior a .40. La estructura final es de una sola dimensión, lo cual explica $57 \%$ de la varianza, con indicadores de bondad de ajuste $\mathrm{Ji}^{2}=20.89, \mathrm{gl}=5, \mathrm{p}=.001$, y convergió en cuatro iteraciones, con valores de $\mathrm{KMO}=.830$, que reflejan que el tamaño de la muestra es adecuado para hacer el análisis factorial. Como se puede observar en la tabla 4, los reactivos hacen referencia, en general, a conductas de rechazo o alejamiento hacia el personaje con discapacidad presentado en la viñeta.

\begin{tabular}{l}
\hline Tabla 4. Estructura factorial de la escala de conductas \\
\begin{tabular}{|l|c|}
\hline & Conductas \\
\hline Alfa de Cronbach & 0.861 \\
\hline Varianza explicada & $57 \%$ \\
\hline Levantarse y salir & .856 \\
\hline Encontrar una excusa para salir & .794 \\
\hline Alejarse & .781 \\
\hline Cambiarse de mesa & .688 \\
\hline Leer el periódico o hablar por un teléfono celular & .636 \\
\hline
\end{tabular}
\end{tabular}

Fuente: elaboración propia. 
Para analizar las relaciones de todas las dimensiones identificadas como elementos subyacentes al constructo más general de actitudes hacia personas con discapacidad, se llevó a cabo un análisis factorial confirmatorio mediante el programa AMOS 22 (Arbuckle, 2013). Para este tipo de análisis, Jackson (2003) propone una proporción óptima de 20 casos por cada parámetro a estimar, que para este modelo son 20 (12 cargas, 1 covarianza y 7 varianzas), lo que resulta en un tamaño de muestra estimado ideal de 200 casos.

En general, los resultados muestran buenos indicadores de bondad de ajuste y se observan discrepancias no significativas entre las relaciones planteadas en el modelo confirmatorio y las observadas en los datos analizados $\left(\mathrm{Ji}^{2}=7.66, \mathrm{gl}=3, \mathrm{p}=.053\right)$. Con un valor de $\mathrm{RMR}=.022$, se puede concluir que resta poca varianza compartida entre los reactivos una vez que se extrae la varianza explicada por las variables latentes. También se señala un adecuado ajuste del modelo. El indicador GFI=.984, el cual toma valores entre 0 y 1 , señala niveles elevados de varianza total explicada por medio del modelo teórico; mientras que el índice $\mathrm{CFI}=.98$, con valores también de 0 a 1 , señala diferencias sustanciales ente el modelo propuesto y un modelo hipotético de relaciones nulas entre los elementos analizados. Los valores de CFI y GFI por encima de .95 denotan un adecuado ajuste entre el modelo y los datos analizados (Levy y Varela, 2003; Brown, 2006). El indicador RMSEA, que estima la estabilidad de la bondad de ajuste esperada del modelo al trabajar con la población de la misma muestra, refleja un buen índice (por debajo de .08), sólo en su intervalo de confianza más bajo (RMSEA=.090, intervalo de confianza a $90 \%$ bajo<.001, alto=.171), lo cual señala que el ajuste observado podría ser diferente con la población en su conjunto. Todas las relaciones que se muestran en la figura 1 son estadísticamente significativas, con valores de C.R. mayores a -2.86 y valores de $p<004 p a r a$ todos los casos, lo que indica que todos los reactivos son relevantes en la configuración de la varianza común en la variable latente.

Estas cargas pueden verse afectadas por muestras pequeñas que reducen la potencia del análisis (Kline, 2011), por lo que estos resultados señalarían un tamaño de muestra suficiente para identificar efectos significativos para cada reactivo. Estos efectos deben considerarse con reserva cuando son presentados de manera estandarizada como extrapolaciones de la muestra hacia la población en general (Kline, 2011). Por esta razón, considerando que el muestreo no es representativo, en la figura 1 se presentan solo los puntajes de efectos no estandarizados. Como se observa en la figura 1, las relaciones con el constructo general son positivas para las conductas y las emociones, y negativas para las cogniciones, lo cual es conceptualmente congruente, puesto que las cogniciones refieren cercanía y expectativas positivas de la interacción, mientras que conductas y emociones refieren aspectos negativos. Para la variable latente Emociones, se observan relaciones positivas con Ansiedad y Prejuicio, y negativas con Tranquilidad, lo que refleja el contenido conceptual opuesto de Tranquilidad con los otros dos componentes.

Analizando las relaciones residuales, se identificó una covariación positiva entre el indicador de Tranquilidad y el de Cogniciones, lo cual indica que la tranquilidad emocional y las cogniciones que hacen referencia a expectativas positivas hacia la interacción con una persona con discapacidad se asocian positivamente, aunque no pueden establecerse - al menos desde estos datos - relaciones causales entre ellas. Esta relación puede derivarse del hecho de compartir ambos elementos una valencia positiva hacia las personas con discapacidad, mientras que el resto de las dimensiones implicarían valoraciones negativas. 


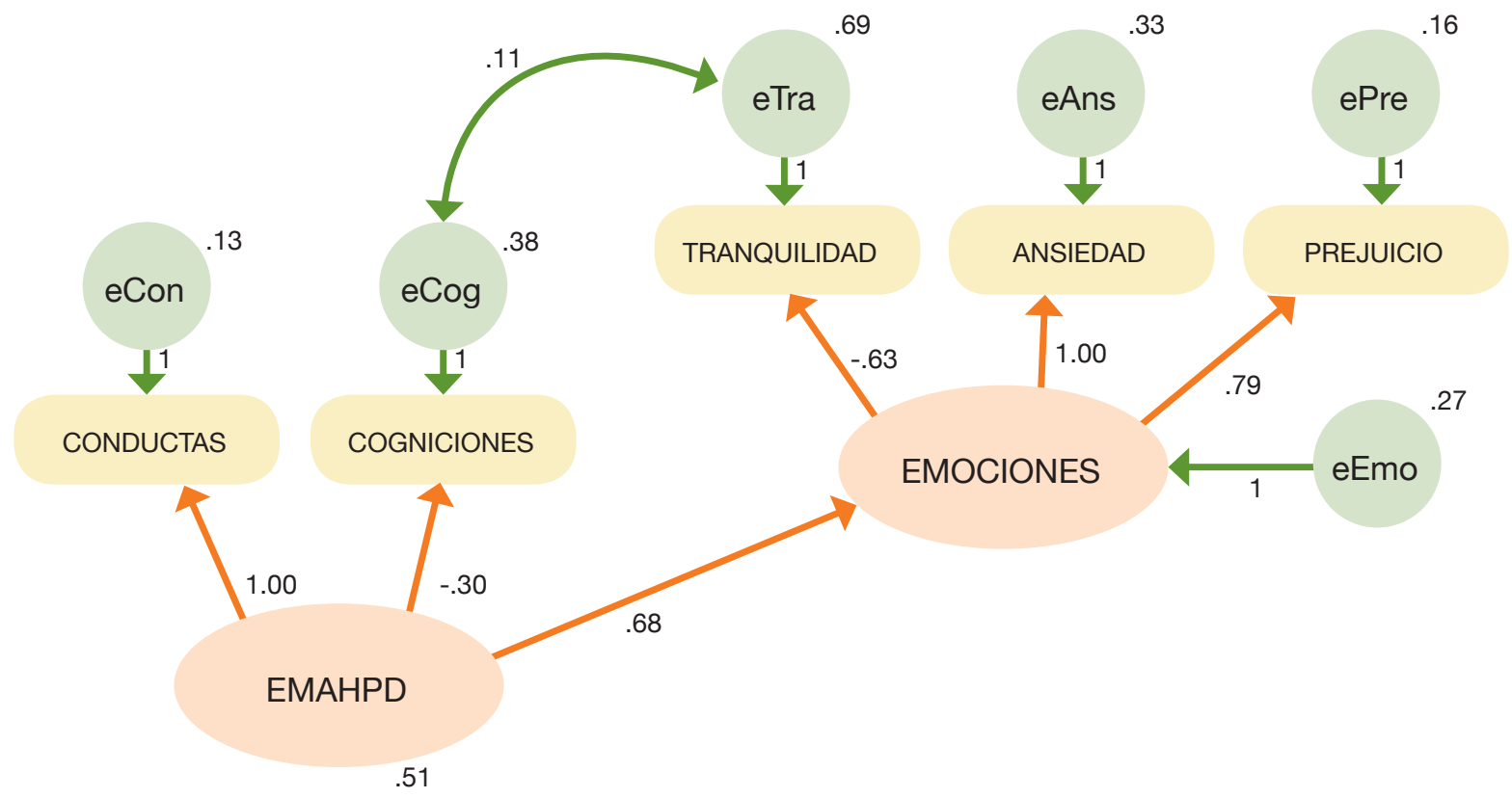

Fuente: elaboración propia.

Respecto a la medición de calidez y competencia, los reactivos fueron sometidos a un análisis factorial de máxima verosimilitud con rotación oblicua directa. Los resultados señalan que una solución convergió en cuatro rotaciones, lo que explicó en conjunto $61,54 \%$ de la varianza mediante dos factores con niveles de consistencia interna superiores a .820. Esta solución es consistente con la encontrada previamente por Fiske et al. (2002), tanto en la estructura de dos dimensiones como en los niveles adecuados de consistencia interna. Como se muestra en la tabla 5, el primer factor corresponde a la dimensión de calidez, mientras que el segundo corresponde, en teoría, a la dimensión de competencia. Debe señalarse el caso del reactivo Inteligente, el cual, teóricamente, formaba parte de la dimensión de competencia, pero para esta muestra en particular se agrega en el factor de calidez. Los valores de $\mathrm{KMO}=.882$ denotan que el tamaño de la muestra fue suficiente para realizar este análisis. 


\begin{tabular}{|c|c|c|}
\hline & Calidez & Competencia \\
\hline Alfa de Cronbach & .915 & .821 \\
\hline Varianza explicada & $50.04 \%$ & $11.50 \%$ \\
\hline Tiene buenas intenciones & .907 & -.082 \\
\hline Sincera & .844 & -.092 \\
\hline Tiene buena voluntad & .834 & -.030 \\
\hline Confiable & .752 & .053 \\
\hline Cálida & .683 & .025 \\
\hline Amistosa & .678 & .226 \\
\hline Inteligente & .543 & .347 \\
\hline Hábil & -.153 & .791 \\
\hline Eficiente & .085 & .727 \\
\hline Competente & .102 & .678 \\
\hline Capaz & .113 & .668 \\
\hline
\end{tabular}

Fuente: elaboración propia.

\subsection{Pruebas de hipótesis}

Como puede observarse en la tabla 6, los puntajes reportados muestran en general niveles bajos tanto de actitudes negativas hacia las personas con discapacidad, como en los factores Prejuicio y Conductas, ambos con una connotación negativa, y puntajes por encima de la media teórica (5) en los factores Cognición, Calidez y Competencia, los tres con implicaciones positivas hacia las personas con discapacidad. Los factores Ansiedad y Tranquilidad muestran un patrón menos claro, dado que ambos - conceptualmente opuestos - cruzan la media teórica. Al analizar los reactivos que conformaron la escala de Cognición, el reactivo con puntuación más alta fue "¿Por qué no llegar a conocerlo mejor" ( $\bar{x}=4.21)$, mientras que en la escala de Conducta, en los reactivos: "Cambiarse de mesa" ( $\bar{x}=2.26)$ y "Leer el periódico o hablar por teléfono" $(\bar{x}=2.26)$ se obtuvieron las puntuaciones más altas; en cambio la puntuación más baja fue en "Levantarse y salir" ( $\overline{\mathrm{X}}=1.69)$.

Se consideró probable que estas percepciones se vieran modificadas por la cercanía de los participantes con personas con discapacidad. Por ello, se obtuvo información respecto al tipo de contacto previo con este tipo de personas: 83,6\% declararon conocer a alguna otra persona con alguna discapacidad. De quienes conocen a personas con discapacidad, en la mayoría de los casos (40\%) resultó ser parte de su familia, el $38 \%$ declaró sólo conocerla y el $18 \%$ dijo tener una relación de amistad.

La discapacidad más frecuente de las personas conocidas es la motriz (56,9\%), seguida de la intelectual $(21,6 \%)$ y la visual $(13,7 \%)$. Para poner a prueba esta hipótesis se llevaron a cabo análisis de varianza de una vía. Como variable independiente se tomó el tipo de relación con una persona con discapacidad (es 
decir, no conoce a nadie, conocido, amigo o familiar) y como dependientes los factores de Competencia y Calidez, y los factores de Prejuicio, Ansiedad, Tranquilidad, Cogniciones y Conducta, de la medición de la Escala Multidimensional de Actitudes Hacia Personas con Discapacidad. Los resultados muestran promedios similares entre los diferentes grupos, con lo que se obtuvieron valores de F entre .122 y 1.99, considerados no estadísticamente significativos en todos los casos (valores de p>.133).

Tabla 6. Estadísticos descriptivos de las Escalas Multidimensional de Actitudes Hacia Personas con Discapacidad y de Calidez y Competencia

\begin{tabular}{|l|c|c|c|c|c|c|c|}
\cline { 2 - 8 } \multicolumn{1}{c|}{} & Prejuicio & Tranquilidad & Ansiedad & Cognición & Conductas & Calidez & Competencia \\
\hline Promedio & 1.66 & 2.89 & 2.72 & 3.91 & 1.84 & 4.25 & 4.30 \\
\hline $\begin{array}{l}\text { Desviación } \\
\text { estándar }\end{array}$ & 1.42 & 0.95 & 0.92 & 0.65 & 0.8 & .695 & .624 \\
\hline
\end{tabular}

Fuente: elaboración propia.

Respecto a la asignación de rasgos de Calidez o Competencia a los personajes en las viñetas, se observan puntajes similares $(t=1.54, \mathrm{gl}=182, \mathrm{p}=.125)$ en la percepción de Competencia (discapacidad $\bar{x}=4.29$; $\sin$ discapacidad $\bar{x}=4.22$ ), pero diferencias menores - aunque significativas - en la percepción de Calidez (t=2.90, $\mathrm{gl}=181, \mathrm{p}=.004)$, con puntajes más altos para el personaje con discapacidad $(\overline{\mathrm{x}}=4.24)$, en comparación con el personaje sin discapacidad ( $\bar{x}=4.12$ ); en ambos casos esto refleja una valoración de estereotipos positivos, pero significativamente más altos para la Calidez en el caso del personaje de la viñeta con discapacidad.

\section{Discusión}

El propósito de esta investigación fue analizar las actitudes y estereotipos que existen hacia las personas con discapacidad, además de obtener evidencias de la validez de constructo, criterio y consistencia interna de los instrumentos empleados para dicho fin. En primer lugar, la estrategia de muestreo de este estudio implica una escasa representación para la población en general, pero permite una correcta aproximación a procesos cognitivos centrales de la discriminación en quienes, por ser responsables de la atención de la salud de otros, muy probablemente atenderán a personas con discapacidad en algún momento. Al respecto, aunque se buscó la participación de estudiantes de diversas áreas de la salud en la conformación de la muestra, predominaron los psicólogos. Esto es relevante si se considera que los médicos - a diferencia de los psicólogos - son los profesionales que, en la mayoría de los casos, identifican y proporcionan atención médica a las personas con discapacidad. Además, se ha documentado que en ellos prevalecen actitudes menos positivas hacia este colectivo (López-Ramos, 2004), las cuales pueden influir de manera negativa en el tipo de información y servicio que se les brinda a ellas y a sus familias.

En cuanto al proceso de validación de ambos instrumentos, el análisis factorial muestra que los factores obtenidos guardan una relación estrecha con los supuestos teóricos que sustentan cada instrumento. Asi- 
mismo, explican un porcentaje adecuado de la varianza y cuentan con un índice de confiabilidad adecuado. En suma, se puede afirmar que los instrumentos poseen propiedades psicométricas adecuadas para evaluar los constructos teóricos para los cuales fueron diseñados. Esto es destacable si se considera que existen pocos instrumentos de este tipo adaptados y validados en poblaciones que hablan español, lo cual permite seguir generando investigación de este tipo en países de Latinoamérica (Stevens et al., 2013). Cabe señalar que, a pesar de que el tamaño de la muestra también es reducido para lograr la representatividad muestral, es suficiente para el estudio de procesos cognitivos que se asume que funcionan de manera similar en amplios sectores de la población. Es decir, aunque los promedios absolutos obtenidos en los instrumentos podrían variar al sondear muestras más amplias, se esperaría que las propiedades psicométricas de los instrumentos y el patrón de relaciones entre las variables, principales focos de este estudio, sean equivalentes. De hecho, este tamaño de muestra es similar al utilizado por Fiske et al. (2002) en el estudio que dio origen a la escala de Calidez y Competencia aquí utilizada (185 participantes), además de que se obtuvieron índices que reflejan un adecuado tamaño de muestra. Sin embargo, es necesario reconocer el valor de hacer nuevos estudios en muestras más amplias que permitan representatividad y comparación en diferentes contextos socioculturales.

La Escala Multidimensional de Actitudes Hacia Personas con Discapacidad estuvo constituida por tres componentes que conforman las actitudes: emocional, cognitivo y conductual. A su vez, el componente emocional estuvo integrado por tres factores que representan prejuicio, tranquilidad y ansiedad. En el componente cognitivo se eliminó el reactivo "Es posible que nos llevemos bien". Esto puede deberse a que el reactivo permite más de una interpretación, es decir, mientras los demás reactivos presentan cogniciones positivas hacia la interacción con alguien que tiene discapacidad, éste refiere sólo una posibilidad, dejando abierta la interpretación a que no sea así.

Tanto el análisis exploratorio como el confirmatorio permitieron especificar con mayor detalle la estructura de la dimensión de emociones de la escala. Aunque el modelo teórico proponía una estructura general de sólo tres componentes, los análisis aquí mostrados reflejan que - al menos para esta muestra - el componente emocional estaría integrado por las dimensiones de tranquilidad, ansiedad y prejuicio. Aunque subyacen a una misma dimensión emocional — como se refleja por la adecuada bondad de ajuste del modelo-, los tres componentes tienen también características que los hacen diferentes entre sí. El componente de Prejuicio contiene emociones de rechazo, seguramente derivadas de valoraciones negativas actitudinales hacia las personas con discapacidad. Este componente, así como el de Ansiedad, se asocian positivamente con la dimensión emociones, mientras que el de Tranquilidad presenta una carga positiva hacia el mismo. Así, la dimensión de emociones está definida por una connotación negativa hacia las personas con discapacidad. Estos resultados confirman que, en las relaciones con personas con discapacidad, las conductas están íntimamente relacionadas con las cogniciones o creencias hacia ellas y las emociones que estas creencias generan. Esto enfatiza la relevancia del trabajo de sensibilización en los profesionales de salud con respecto a sus creencias y emociones para que éstas repercutan en sus conductas (Hampton et al., 2007).

Los análisis descriptivos de la escala referida anteriormente permitieron identificar que los participantes tuvieron puntuaciones que indican actitudes positivas hacia las personas con discapacidad, tal y como se muestra en otras investigaciones con profesionales de la salud (Moreno et al., 2006). Cabe recordar que, en este estudio, la muestra estuvo integrada principalmente por profesionales de la psicología, en quienes se han identificado actitudes más positivas hacia este colectivo (Polo et al. 2011). Al llevar a cabo el análi- 
sis con los factores de la escala de emociones, se observa que los participantes obtuvieron la puntuación más baja en Prejuicio, seguido de Ansiedad; la más alta fue en Tranquilidad. Esto sugiere que, aunque las personas no sientan rechazo ni miedo al interactuar con una persona con discapacidad, pueden sentirse ansiosas al desconocer la manera adecuada de interactuar con ella. En cuanto a la escala de Cognición, los participantes obtuvieron puntuaciones que reflejan pensamientos positivos hacia la interacción con una persona que tiene discapacidad; el reactivo con puntuación más alta es “¿Por qué no llegar a conocerlo mejor?", lo cual muestra una disposición a relacionarse con esa persona, tal y como se ha documentado en otras investigaciones (Polo et al., 2011). En cuanto a la escala de Conducta, los participantes obtuvieron puntuaciones bajas en este factor; sin embargo, en los reactivos: "Cambiarse de mesa" y "Leer el periódico o hablar por teléfono" se observan las puntuaciones más altas, mientras que el reactivo con la puntuación más baja fue "Levantarse y salir", lo que sugiere que las personan optan por formas más sutiles de rechazo.

Esta investigación también buscó obtener indicadores de los estereotipos que se manejan hacia las personas con discapacidad en términos de la Calidez y Competencia. Un dato interesante está relacionado con los hallazgos del análisis factorial, en donde el reactivo Inteligente se ubicó en el factor de Calidez, cuando se esperaba que estuviera en el de Competencia. Esto indica que, para esta muestra en particular, la inteligencia es entendida como parte de un grupo de habilidades necesarias para una adecuada socialización y la aceptación de los demás, más que un atributo ligado a la transformación del entorno y el logro de metas.

Los resultados de este instrumento muestran que los participantes otorgan calificaciones altas en Calidez y Competencia a la persona con discapacidad, posiblemente porque tienen una baja disposición a reportar estereotipos de incompetencia hacia este tipo de personas. Asimismo, brindaron puntuaciones más altas en los atributos de Calidez a la persona con discapacidad en comparación con las otorgadas a la persona sin discapacidad, quizá como una percepción compensatoria o posiblemente porque persiste la idea de que las personas con discapacidad son débiles y por ello necesitan la compasión de los demás (Boyle, 2010). Los promedios de los atributos de Calidez y Competencia no permiten pensar en un proceso de estereotipamiento afectivamente negativo, aunque sí diferencial en términos de calidez, el cual puede asociarse a la asignación de papeles de subordinación y menor estatus social, puesto que se les percibe como cálidos en comparación con otras personas sin discapacidad igualmente competentes.

Por último, en este estudio el hecho de que los participantes tuvieran algún tipo de contacto previo con una persona con discapacidad no intervino en las actitudes hacia ellas. Al respecto, es importante señalar que la gran mayoría de los participantes (86\%) conocía a una persona con discapacidad y para el $40 \%$ esa persona era un familiar, en quienes se esperaría - como otros estudios indican (Sánchez et al., 2011) - que tuvieran actitudes aun más positivas derivadas del tipo de relación afectiva característica de los vínculos familiares. Otra posible interpretación es que, dado que la mayoría de los participantes tuvo algún tipo de contacto previo con una persona con discapacidad, es probable que las actitudes positivas reportadas por los participantes sean producto de dicho contacto, tal y como se muestra en otras investigaciones (Meyers y Lester, 2016).

Los resultados de esta investigación muestran la importancia de seguir investigando cómo operan los procesos de perjuicio y discriminación hacia las personas con discapacidad. Tal vez sea necesario llevar a cabo diseños experimentales que permitan simular situaciones en las que se observe de manera directa lo que la gente realmente hace cuando está en contacto con una persona con discapacidad. También resulta ne- 
cesario distinguir entre evaluaciones globales como éstas y evaluaciones respecto a funciones y roles particulares que implican diferencias de estatus. Valdría la pena probar estos modelos en poblaciones menos escolarizadas o en ambientes donde la discriminación y el maltrato es más abierto, por ejemplo, en niños (Voeltz, 1980). Otra alternativa es elaborar o adaptar este tipo de instrumentos para conocer la percepción de las personas con discapacidad respecto a las actitudes y estereotipos que tiene la gente hacia ellos.

Aunque las variables aquí utilizadas, en general, son de corte cognitivo, esto no indica que las posibles intervenciones sólo deban centrarse en presentar información que contradiga los estereotipos tradicionales hacia las personas con discapacidad. Si asumimos - como se vio aquí- que conducta, cogniciones y emociones están íntimamente relacionadas, resulta indispensable desarrollar estrategias en estos ejes.

Asimismo, pese a que en este estudio el tipo de contacto previo no intervino en las actitudes hacia las personas con discapacidad de los participantes, es importante seguir investigando, pues en otros estudios se ha demostrado que los individuos tienen actitudes más positivas hacia las personas con discapacidad cuando las conocen (Satchidanand et al., 2012). Esto puede derivar en modelos de intervención poderosos, como el de contacto intergrupal, los cuales permitan modificar la dinámica de discriminación. Pettigrew y Tropp (2006) proponen que, cuando los individuos entran en contacto con miembros de un grupo social diferente, es posible reducir los prejuicios intergrupales, sobre todo cuando este contacto cumple las condiciones de igualdad de estatus, metas comunes, tareas de cooperación intergrupal y apoyo de las autoridades o agentes reguladores (ejemplo: facilitadores que dirigen la intervención o autoridades en una institución). Para ello, es importante no omitir factores contextuales particulares de los grupos en cuestión, con el fin de diseñar intervenciones idóneas para cada caso (Pettigrew y Hewstone, 2017).

Finalmente, los hallazgos de este tipo de estudios en profesionales de la salud son relevantes para conocer y promover percepciones positivas hacia las personas con discapacidad, pues de ellos dependerá la atención y calidad de los servicios de salud que reciba este colectivo. Asimismo, es imperativo continuar generando nuevas investigaciones que analicen las actitudes y estereotipos hacia las personas con discapacidad, ya que éstas pueden ser clave para su inclusión social. 
Antonak, R. F. y Livneh, H. (2000): "Measurement of attitudes towards persons with disabilities". Disability and Rehabilitation, 22 (5): 211-224.

Antonak, R. F. y Livneh, H. (1988): The Measurement of Attitudes Toward People with Disabilities: Methods, Psychometrics and Scales. Springfield, IL: Thomas.

Antonak, R. F. (1982): "Development and psychometric analysis of the Scale of Attitudes Toward Disabled Persons". Journal of Applied Rehabilitation Counseling, 13: 22-29.

Arbuckle, J. L. (2013): Amos (Versión 22.0) [Programa de cómputo]. Chicago: SPSS.

Boyle, M. J. et al. (2010): "Attitude of undergraduate health science students toward patients with intellectual disability, substance abuse, and acute mental illness: a cross-sectional study". BMC Medical Education, 10 (71): $1-8$.

Brown, T. (2006): Confirmatory Factor Analysis for Applied Research. Nueva York: The Guilford Press.

Carver, C. S. et al. (1978): "Favorable Evaluations of Blacks and the Handicapped: Positive Prejudice, Unconscious Denial, or Social Desirability?". Journal of Applied Social Psychology, 8 (2): 97-106.

Christison, G. W. et al. (2002): "The medical condition regard scale: measuring reactions to diagnoses". Academic Medicine, 77 (3): 257-262.

Consejo Nacional para Prevenir la Discriminación, Conapred (2012): Encuesta Nacional sobre Discriminación en México 2010. Resultados sobre personas con discapacidad (en línea). <http://www.conapred.org.mx/documentos_cedoc/Enadis-2010-RG-Accss-002.pdf>, acceso 24 de mayo de 2018.

Díaz, S. M. y Quiroga, L. G. (2007): “Actitudes de los estudiantes de la salud de la Universidad de Chile hacia las personas con discapacidad”. Revista Chilena de Terapia Ocupacional, (7): 3-12.

Feinberg, L. B. (1967): "Social desirability and attitudes toward the disabled". Journal of Counseling \& Development, 46 (4): 375-381.

Ferguson, L. T. (1970): "Components of attitudes toward the deaf". Proceedings of the Annual Convention of the American Psychological Association, 5: 693-694.

Findler, L. et al. (2007): "The Multidimensional Attitudes Scale Toward Persons with Disabilities (MAS): Construction and validation”. Rehabilitation Counseling Bulletin, 50: 166-176.

Fiske, S. T. et al. (2002): "A model of (often mixed) stereotype content: competence and warmth respectively follow from perceived status and competition”. Journal of Personality and Social Psychology, 82 (6): 878.

Gething, L. y Wheeler, B. (1992): "The Interaction With Disabled Persons Scale: A new Australian instrument to measure attitudes towards people with disabilities". Australian Journal of Psychology, 44: 75-82.

Goodwin, K.A., y Goodwin, C.J. (2017): Research in Psychology: Methods and Design (8 ${ }^{\text {th }}$ ed.). Nueva York: Wiley.

Grand, S. A. et al. (1982): "Attitudes toward disabled persons as a function of social context and specific disability”. Rehabilitation Psychology, 27: 165-173.

Hampton, N. Z. y Xiao, F. (2007): "Attitudes toward people with developmental disabilities in Chinese and American students: The role of cultural values, contact, and knowledges”. Journal of Rehabilitation, 73 (4): 23-32. 
Henson, R. K. y Roberts, J. K. (2006): "Use of exploratory factor analysis in published research: Common errors and some comment on improved practice". Educational and Psychological measurement, 66 (3): 393-416.

Hodge, S. et al. (1998): Validation of the Physical Educators' Perceptions of Inclusion Inventory-Revised. Manuscrito no publicado.

Instituto Nacional de Estadística y Geografía, INEGI (2015): Estadísticas a propósito del día internacional de las personas con discapacidad (3 de diciembre) (en línea). <http://www.inegi.org.mx/saladeprensa/aproposito/2015/discapacidad0.pdf>, acceso 24 de mayo de 2018.

Jackson, D. L. (2003): "Revisiting sample size and number of parameter estimates: Some support for the N:q hypothesis". Structural Equation Modeling, 10: 128-141.

Kaiser, H. F. (1970): “A second generation Little Jiffy”. Psychometryka, 35 (4): 401-415.

Kline, R. (2011): Principles and practice of structural equation modelling. Nueva York: The Guilford Press.

Levy, J. y Varela, M. (2003): Análisis multivariable para las Ciencias Sociales. Madrid: Pearson Prentice Hall.

López-Ramos, V. M. (2004): Estudio sobre las actitudes hacia las personas con discapacidad entre los estudiantes de la Universidad de Extremadura (tesis doctoral). Universidad de Extremadura, España.

MacCallum, R. C. et al. (2001): "Sample size in factor analysis: The role of model error". Multivariate Behavioral Research, 36: 611-637.

Mackelprang, R. W. y Salsgiver, R. O. (1996): "People with disabilities and social work: Historical and contemporary issues". Social Work, 41 (1): 7-14.

Makas, E. et al. (1988): "The issues in disability scale: A new cognitive and affective measure of attitudes toward people with physical disabilities". Journal of Applied Rehabilitation Counseling, 19: 21-29.

Marini, I. (2012): "Societal attitudes and myths about disability", en Marini, I et al. (eds.): Psychosocial Aspects of Disability: Insider Perspectives and Strategies for Counselors. Nueva York: Springer.

Martínez et al. (2011): "Prejuicios entre los estudiantes hacia las personas con discapacidad: Reflexiones a partir del caso de la universidad de Alicante". Alternativas, 18: 75-90.

Mella, S. y González, L. (2007): "Actitudes de los estudiantes de la salud de la Universidad de Chile hacia las personas con discapacidad". Revista Chilena de Terapia Ocupacional, 7: 1-14.

Meyers, S. y Lester, D. (2016): “An attempt to change college students' attitudes toward individuals with disabilities". Comprehensive Psychology, 5: 1-7.

Moreno, F. J. et al. (2006): "Actitudes ante la discapacidad en el alumnado universitario matriculado en materias afines". Revista Iberoamericana de Educación, 40 (5): 1-12.

Novo-Corti, I. et al. (2015): "Los futuros docentes y su actitud hacia la inclusión de personas con discapacidad. Una perspectiva de género". Anales de Psicología, 31 (1): 155-171.

Organización Mundial de la Salud, OMS (2015): Discapacidad y salud (en línea). <http://www.who.int/mediacentre/ factsheets/fs352/es/>, acceso 24 de mayo de 2018.

Organización Mundial de la Salud, OMS (2011). Informe mundial sobre la discapacidad (en línea). <http://www. who.int/disabilities/world_report/2011/accessible_es.pdf?ua=1>, acceso 24 de mayo de 2018. 
Organización Mundial de la Salud, OMS (2001): Clasificación Internacional del Funcionamiento, de la Discapacidad y de la Salud (en línea). <http://apps.who.int/iris/bitstream/10665/43360/1/9241545445_spa.pdf>, acceso 24 de mayo de 2018.

Pettigrew, T. F. y Hewstone, M. (2017): "The Single Factor Fallacy: Implications of Missing Critical Variables from an Analysis of Intergroup Contact Theory1". Social Issues and Policy Review, 11 (1): 8-37.

Pettigrew, T. F. y Tropp, L. R. (2006): "A meta-analytic test of intergroup contact theory”. Journal of Personality and Social Psychology, 90 (5): 751-783.

Polo, M.T. et al. (2011): "Estudio de las actitudes de estudiantes de Ciencias Sociales y Psicología: relevancia de la información y contacto con personas discapacitadas". Universitas Psychologica, 10 (1): 113-123.

Satchidanand et al. (2012): "Attitudes of healthcare students and professionals toward patients with physical disability". American Journal of Physical Medicine \& Rehabilitation, 91 (6): 533-545.

Siller, J. y Braden, B. (1976): "A factor analytically derived scale to measure attitudes toward cancer”. Nueva York: New York University School of Education.

Siller, J. et al. (1967a): "Attitudes of non-disabled toward the physically disabled", en: Studies in Reactions to Disability XI. Nueva York: School of Education at New York University.

Siller, J. et al. (1967b): "Structure of attitudes toward the physically disabled: The Disability Factor Scales Amputation, Blindness, Cosmetic Conditions", en Studies in Reactions to Disability XII. Nueva York: New York University School of Education.

Stevens et al. (2013): "Factor analysis of the Spanish multidimensional attitudes scale toward person with disabilities". American Psychological Association, 58 (4): 396-404.

Tervo, R. C. et al. (2002): "Medical students' attitudes toward person with disability: A comparative study". Archives of Phsysical Medicine and Rehabilitation, 83: 1537-1542.

Tringo, J. L. (1970): The hierarchy of preference toward disability groups. Journal of Special Education, 4: 295-306.

Vann, D. H. (1970): "Components of attitudes toward the obese including presumed responsibility for the condition". Proceedings of the Annual Convention of the American Psychological Association, 5: 695-696.

Verdugo, M. A. et al. (1994): Actitudes hacia las personas con minusvalía. Madrid: Ministerio de Asuntos Sociales, Instituto Nacional de Servicios Sociales.

Voeltz, L. M. (1980): “Children's attitudes toward handicapped peers”. American Journal of Mental Deficiency, 84: $455-464$

Yuker, H. E. y Hurley, M. K. (1987): "Contact with and attitudes toward persons with disabilities: The measurement of intergroup contact". Rehabilitation Psychology, 32 (3): 145.

Yuker, H. E. et al. (1960): A scale to measure attitudes toward disabled persons Human Resources Studies). Albertson, NY: Human Resources Foundation. 